\title{
EVALUACIÓN DE LOS PROGRAMAS DE FORMACIÓN OCUPACIONAL PARA EL COLECTIVO DE MUJERES
}

\section{[Evaluation of Occupational Training Programs for women]}

por

$\underline{\text { Article record }}$

$\underline{\text { About authors }}$

HTML format
Ficha del artículo

Inmaculada Chiva

(ichiva@uv.es) $\underline{\text { Sobre los autores }}$

Formato HTML

\section{Abstract}

This paper summarizes the results obtained from the evaluation of Occupational Training Programs through which we checked the quality of service and the efficiency of the introduction of women into the labour market, as well as the need to promote quality Occupational Training specifically for women that will not only facilitate their introduction into the labour market by adapting their personal skills to its needs, but will also encourage personal and social improvement.
\end{abstract}

\section{Keywords}

Evaluation, occupational training, women, quality, labour market.

\begin{abstract}
Resumen
Este artículo trata de resumir los resultados obtenidos en la evaluación de los programas de Formación Ocupacional para comprobar la calidad del servicio y la eficacia en el logro de la inserción laboral del colectivo de mujeres. De esta manera, hemos comprobado la necesidad de seguir promoviendo una Formación Ocupacional específica de calidad para la mujer ya que no sólo le va a permitir su inserción en el mercado laboral, adaptando sus cualificaciones a las necesidades del mercado de trabajo, sino que también le va a suponer una mejora personal y social.
\end{abstract}

\section{Descriptores}

Evaluación, formación ocupacional, mujeres, calidad, inserción laboral.

\section{Introducción}

Los estudios e investigaciones realizadas en estos últimos años han destacado el cambio de una sociedad industrial a una sociedad postindustrial, lo que ha conllevado un cambio en el mercado laboral traducido en las siguientes características (adaptado de Pedraza, 2001):

- Globalización/mundialización de la economía.

- Nueva organización del trabajo.

- Animación ocupacional (que como veremos más tarde no es suficiente, sobre todo en ciertos colectivos). 
- Aumento de trabajadores/as terciarios y disminución de los dedicados a la producción.

- Concreción de la inteligencia, del conocimiento, de los factores soportantes de la producción.

- Cambio de la estructura profesional concentrada en los profesionales, científicos y técnicos.

- Innovaciones tecnológicas que se desarrollan como consecuencia de investigaciones y aplicaciones en el campo de la informática y de las biotecnologías. Sociedad de la Información.

- Ampliación de los sectores sociales en el nuevo contexto social, como son los técnicos y científicos.

- Crecimiento de la tasa de escolaridad de las fuerzas de trabajo.

- Dinamicidad de las fuerzas de trabajo femeninas.

- Elevada tasa de inocupación juvenil...

Esto supone que el aumento del empleo, en estos últimos 20 años, se ha dado en el sector servicios, exigiéndose una mayor cualificación, dándose una disminución de empleos para trabajadores/as de escasa o nula cualificación.... Asociado a esto, en la economía actual priman la productividad, la innovación y la competitividad, lo que implica nuevos requisitos de competencias y cualificaciones para los trabajadores/as, donde se promete un trabajo más global y autónomo, y una mayor participación del trabajador/a en la planificación y el control del proceso productivo (Pedraza, 2001). Se considera, por tanto, al factor humano como recurso central de la empresa junto al financiero, por lo que ésta se estructura cada vez más en función del saber y de las competencias de sus trabajadores/as.

Sin embargo, podemos apuntar que el crecimiento económico operado en los últimos años en las sociedades europeas y los cambios laborales producidos, se han manifestado de manera muy frágil para los colectivos de más difícil acceso o inserción en el mercado de trabajo. Además, buena parte de las medidas de apoyo a la generación de empleo han sido utilizadas la mayoría de veces por personas que probablemente hubieran accedido al mercado de trabajo sin necesidad de las mismas. Esto ha producido que los desequilibrios se refuercen, y que sea necesario establecer prioridades selectivas en las medidas de apoyo para que puedan conseguirse mayores cotas de inserción por parte de estos colectivos. Esto nos conduce a afirmar que se deben de potenciar las políticas de formación, y no apostar por la precariedad/inestabilidad laboral sobre todo de los colectivos más desfavorecidos.

Podemos destacar que existen colectivos con mayores dificultades que son considerados por los poderes públicos como especialmente merecedores de protección de cara a su formación y/o acceso al mercado de trabajo entre ellos nos encontramos a: mujeres, jóvenes, parados/as mayores de 40 años (podemos encontrar más información acerca de las características del colectivo de parados mayores de 40 años en Chiva, 2005) ... Esta distinción es pertinente puesto que no se pueden diseñar políticas concretas de inserción de forma unívoca, debido a que el paro no es un fenómeno estructural que se distribuye de manera uniforme sino que afecta de manera desigual a los diferentes grupos.

En cuanto al colectivo de mujeres, colectivo objeto de estudio y uno de los más castigados, podemos afirmar que empiezan a participar en el mercado laboral de forma masiva y continua a partir del cambio de valores que se produce en el año 75 y a partir de la nivelación de oportunidades que la educación produce entre los dos sexos. Esta situación supone que el colectivo femenino pasa a ser un colectivo bien cualificado, más polivalente, con más capacidad de adaptación a los nuevos mercados laborales. Sin embargo, es un colectivo que tiene dificultad de inserción laboral por repartir su tiempo de trabajo con tareas domesticas familiares y dedicar menos tiempo a procesos de búsqueda y actualización profesional. Por tanto, el paro, la ocupación a tiempo parcial y, especialmente la 
inactividad son situaciones que afectan a una mayor proporción de mujeres que de hombres (Indicadores Sociales de España 2003, INE).

Ante está situación se obtiene una voz que aclama que la Formación Profesional juega y jugará un papel básico en el progreso económico y social de los países, y en la mejora de las condiciones laborales de las mujeres.

Dentro de este marco, la Unión Europea a través del Fondo Social Europeo, junto con los gobiernos nacionales y regionales, organiza actividades orientadas a la formación para el empleo (en Chiva, 2003 aparece una revisión de los distintos programas, ayudas... que desde la Unión Europea se han implementado a favor de este colectivo) como medio al servicio de la inserción social y laboral, de la adquisición de cualificaciones, para el desarrollo económico y la dinamización del territorio... La implicación de las distintas instituciones y entidades en la organización y desarrollo de esta actividad, genera un panorama de actuación políticoadministrativo complejo a la hora de realizar un seguimiento y evaluación.

En el contexto español, y concretamente en la Comunidad Valenciana, se considera desde hace unos años a la Formación Ocupacional y Continua como un instrumento eficaz de dinamización del empleo. Esta consideración ha generado un proceso de revisión y reforma del sistema de Formación Profesional gestionado por las Administraciones Públicas, para dar respuesta a las necesidades de modernización e incremento de la competitividad del sistema productivo, atender a las demandas de cualificación de la población activa, e implementar las medidas adoptadas por la Unión Europea en este tema.

En la Comunidad Valenciana (contexto en el cual se centra el estudio que aquí se presenta), en 1993 las competencias en materia de Formación Profesional Ocupacional fueron traspasadas por el Estado a la Consellería de Trabajo y Asuntos Sociales de la Genera- litat Valenciana. En ese momento, se propusieron introducir cambios en la gestión de la Formación Ocupacional, al objeto de mejorar su calidad. Esta mejora supuso el incremento del control de las Acciones Formativas, con una racionalización en cuanto a su gestión, una mejora en su desarrollo y un mayor control de la calidad de las mismas. En la actualidad todavía continua vigente un sistema similar de seguimiento y control del desarrollo de las distintas acciones formativas como valoración de la calidad docente.

En el siguiente punto se pretende analizar, más pormenorizadamente, las características que definen a las mujeres tanto en el mercado de trabajo como en la oferta formativa dirigida a este colectivo.

\section{Características de Empleo y For- mación referidas a las mujeres}

"El cambio experimentado en el mercado laboral y en la sociedad en general, en estos últimos años, ya tratado anteriormente, convierte a la mujer en un sujeto social privilegiado y, de sector débil, con un papel protagonista en la nueva sociedad postindustrial" (Battistone, 92:36).

Desde esta perspectiva de cambio laboral, se ha producido en los años 90 la mayor cuota de ocupación por la componente femenina, aunque sin menospreciar una infrautilización de la fuerza de trabajo de las mismas. Esto significa que los elevados incrementos en las tasas de ocupación y de desocupación son fenómenos estrechamente interconectados en el ámbito del mercado de trabajo femenino. Esto se debe a que la incorporación laboral se da con un retraso milenario, con una distancia inabarcable respecto a los hombres. Además la mayor parte de las mujeres han asistido a su incorporación al trabajo sin dejar de asumir como naturalmente propias las responsabilidades familiares del cuidado de los hijos y de la organización de la casa, como si tales funciones fueran absolutamente femeninas y el trabajo remunerado o el ejer- 
cicio de una profesión fuera una actividad que se suma a la propia e indiscutida de "sus labores" (Arce, 1999).

La ausencia de una real igualdad de oportunidades para las mujeres, a pesar de los logros parciales de los últimos años, supone uno de los mayores obstáculos para la construcción de una sociedad basada en los principios de reparto y conciliación de la vida personal, familiar y profesional. Este ha sido el objetivo prioritario de las sucesivas Conferencias Internacionales sobre Mujeres propiciadas por Naciones Unidas: Méjico 1975, Copenhague 1980, Nairobi 1985 y Beijing 1995, donde se han venido reafirmando los derechos de las mujeres y configurando las líneas de actuación política de los gobiernos.

Además, es importante apuntar que para que se produzca un cambio real en la sociedad, partiendo de la igualdad de oportunidades para las mujeres y hombres en todos los sentidos, es necesario actuar desde las bases de la educación y de la estructura familiar a fin de conseguir que las mujeres logren alcanzar a los hombres tanto a nivel formativo como laboral.

\section{Mujeres y Mercado Laboral}

¿Por qué el trabajo de las mujeres está todavía limitado, no sólo por sectores productivos sino también por áreas de mercado y estructuras empresariales? La contestación a la pregunta pasaría por analizar una serie de factores (Battistone, 1992):

- factores culturales que llevan a ver el trabajo femenino independiente como actividad en parte adicional, en parte inestable $y$ arriesgada,

- la escasa escolaridad en capas ancianas,

- el limitado acceso a las estructuras de financiación pública y privada,

- la carencia de estructuras de apoyo para la activación, el despegue y el desarrollo de la empresa,
- la escasa presencia del sector femenino tanto en los trabajos independientes y autónomos como en el mundo empresarial, - ...

En general, parece que la fuerza de trabajo femenina está considerada como fuerza sustitutiva o concurrente de la masculina, más que como fuerza de trabajo adicional. No obstante, hay mercados, ocupaciones y profesiones que parecen abrirse y privilegiar la mano de obra femenina, mientras van desapareciendo viejos prejuicios que tendían a excluir a la mujer de algunas actividades. Sin embargo, estas transformaciones no han causado el efecto de desaparición del fenómeno de la infraocupación y segregación ocupacional femenina.

Esto supone considerar que los aspectos del trabajo femenino no han cambiado tanto con el paso del tiempo, pues existe (Picchio, 1994):

a) una apreciable proporción de mujeres en edad laboral que no tienen un trabajo remunerado,

b) un gran volumen de trabajo doméstico y familiar que realizan las mujeres, empleadas y no empleadas y

c) una concentración de las mujeres en los sectores más pobres de la población trabajadora.

Pasemos a tratar en profundidad los aspectos b) y c), como razones que pueden estar incidiendo en la proporción de mujeres que no tienen un trabajo remunerado pues, muchas de ellas: retornan al trabajo después de educar a sus hijos, afrontan solas la responsabilidad de cuidar a los hijos, reciben insuficiente remuneración de los trabajos para afrontar la manutención y educación de los hijos...

En cuanto al gran volumen de trabajo doméstico y familiar, partimos de que este trabajo es el trabajo asignado en y para la familia y, por tanto, gratuito, que comprende tareas y obligaciones asociadas a la reproducción, cuidado de la casa, tareas burocráticas, 
tareas asistenciales.... La actividad laboral de la mujer no es explicable si no se tiene en cuenta su relación con la esfera de actividades domesticas que realiza en el seno familiar, con el rol familiar que se anticipa a través de diversos momentos de socialización en el rol femenino (Bianchi, 1994). Por ello, reviste una gran importancia el status matrimonial en la comprensión de las relaciones entre formación y empleo. Las diferencias en el tiempo disponible de maridos y esposas para su formación o dedicación laboral son sólo un ejemplo de la incidencia diferencial entre estar o no casada (Izquierdo y otros, 1988). Mientras la paternidad acentúa la legitimidad masculina para su dedicación profesional, la maternidad cuestiona, desde estos parámetros, el elemental derecho al trabajo de la madre. Así "la falta de servicios asequibles al cuidado de los niños y de las niñas, así como otro tipo de iniciativas para hacer compatibles las responsabilidades del cuidado y la educación de los niños y niñas con el empleo, la educación y la formación de los progenitores, constituyen un importante obstáculo para que las mujeres puedan acceder al mercado de trabajo y participen en el mismo en igualdad de oportunidades que los hombres" (Martín Arribas, 1993:10).

Respecto a la concentración de las mujeres en ciertos sectores y profesiones y que además tienen niveles retributivos más bajos y con cualificaciones inferiores, podemos decir que las características que llevan a la mujer a trabajar en estos sectores se deben a la transmisión, recuperación y reelaboración de los contenidos tradicionales de la producción femenina (Martín Arribas, 1993).

Para finalizar con este apartado, y partiendo de las características y dificultades que presenta la mujer ante el mercado laboral, vamos a destacar las afirmaciones más importantes que describen lo que es su trabajo (Comisión Europea, 1996):

a) Las mujeres realizan un trabajo diferente al de los hombres: tal y como hemos visto anteriormente, las mujeres si- guen concentradas en unos pocos sectores económicos: los servicios domésticos, la salud y la educación. Un número creciente de mujeres son activas en el ámbito de ciertos sectores productivos, como en derecho, medicina y contabilidad, pero su presencia todavía no se refleja en los niveles directivos más altos.

b) Las mujeres corren un mayor riesgo de desempleo: como veremos a continuación la tasa de desempleo de las mujeres es superior a la de los hombres.

c) Las mujeres no están superando el “tope invisible": en todos los sectores las mujeres ocupan puestos de trabajo a niveles inferiores, incluso cuando constituyen la mayoría de mano de obra. Las mujeres raramente ocupan puestos de poder y decisión, ocupan una mayor proporción de cuadros medios.

d) Las mujeres ganan menos: la diferencia en los trabajos manuales respecto a los hombres es del 15 al 35\% y en los trabajos no manuales entre el 30 y el $40 \%$. Esta diferencia entre trabajos manuales y no manuales se debe a que en estos últimos las escala de puestos de trabajo es más limitada y los niveles de salario, por tanto, son menos variados.

e) Muchas trabajan jornada parcial: más del $30 \%$ de las mujeres trabajan jornada parcial, ya que permiten a estas combinar el empleo con el cuidado de las personas dependientes. No obstante estos puestos de trabajo, a menudo, proponen bajos salarios, poca cualificación y condiciones de trabajo menos favorables.

f) Las mujeres están creando nuevos puestos de trabajo: en algunos estados europeos las mujeres han creado más del $25 \%$ de las nuevas empresas pequeñas y medianas. Para algunas la creación de una empresa supone una solución al desempleo, pero para otras supone abandonar sus puestos como cuadros medios para dirigir su propia PYME.

A pesar de estas limitaciones la presencia femenina en el mercado de trabajo se ha 
convertido en una realidad cuantitativamente consistente pero que todavía tiene límites que podrían ser subsanados con una buena política formativa a favor de la mujer, unos buenos instrumentos financieros e informativos que funcionaran. Por ello, es importante comprender que todavía el camino para la igualdad entre hombres y mujeres es muy largo y sinuoso.

La situación descrita anteriormente se constata con los datos que aportamos a continuación sobre las tasas de actividad y de paro que presenta la Unión Europea, España y Comunidad Valenciana.

Cifras que concretan la situación de las mujeres en el mercado laboral.

- Las mujeres son uno de los grupos que presentan tasas de actividad más bajas comparadas con las tasas que presentan los hombres, tanto en Europa, como en España y Comunidad Valenciana. Podemos añadir, si observamos la Tabla 1, que las mujeres en Europa superan el 60\% de tasa de actividad y en España y Comunidad Valenciana se acercan al 47\%, mientras que los hombres sobrepasan el $77 \%$ en Europa, y en España y Comunidad Valenciana se acercan al 70\%. Si comparamos la evolución producida desde 1996 al 2005 podremos observar que en el caso de las mujeres ha aumentado la tasa de actividad en los tres contextos, en contraposición los hombres se mantienen con porcentajes similares en Europa, con un ligero aumento en España y Comunidad Valenciana. Esto nos lleva a afirmar que la posición de la mujer en cuanto a la actividad laboral va ganando posiciones con el paso de los años a mayor velocidad que el hombre. Sin embargo, a las mujeres todavía les queda mucho camino por recorrer hasta alcanzar la tasa de actividad de los hombres.

- Las tasas de paro (ver Tabla 2), tanto en Europa, España y Comunidad Valenciana, de las mujeres son siempre superiores a las de los hombres. En el caso europeo se mantiene en un $10 \%$ en las mujeres frente a un $7 \%$ en el caso de los hombres. Por otro lado, en el caso de España y Comunidad Valenciana la diferencia entre ambos grupos es más acusada, ya que podemos encontrarnos distancias de más de 5 puntos de diferencia entre hombres (7\%) y mujeres (12\%). Si comparamos el año 1996 con el 2005 se produce un gran descenso en la tasa de paro, en mayor medida en el caso de las mujeres que en los hombres y también más apreciable en el caso español (se pasa de un 29,56\% a un $12,22 \%$ en el caso de las mujeres y de un $17,60 \%$ a un $7,29 \%$ en el caso de los hombres) y en el caso valenciano (se pasa de un $30 \%$ a un $12 \%$ en las mujeres y de un $16,6 \%$ a un $7,31 \%$ en el caso de los hombres). Todo este análisis nos lleva a afirmar que el descenso producido en las tasas de paro se ha dado en mayor medida en las mujeres, pero todavía se mantiene en tasas superiores que los hombres. Por tanto la distancia que le toca recorrer a las mujeres es todavía bastante grande, sobre todo a nivel estatal y autonómico.

Podemos apuntar que este aumento en la tasa de actividad y el consecuente descenso en la tasa de paro, si comparamos el año 1996 con el 2005, más acusado en el caso de las mujeres, se debe a la mejora económica, laboral y social producida en estos últimos años en toda Europa. Mejora producida, entre otros aspectos, por la implantación de adecuadas políticas activas de empleo, con la puesta en marcha de la Estrategia Europea para el empleo (1997), cuyos resultados se pueden apreciar en estos análisis de tasas de actividad y de paro. Sin embargo no debemos de olvidar que esta mejoría trasladada a ciertos grupos que se encuentran en peor situación económica, social y laboral, como pueden ser las mujeres, todavía no ha alcanzado sus valores óptimos y por tanto es necesario seguir en esta línea. 
Chiva, I. (2006). Evaluación de los programas de formación ocupacional para el colectivo de mujeres.

RELIEVE, v. 12, n. 1, p. 49-74. http://www.uv.es/RELIEVE/v12n1/RELIEVEv12n1_3.htm

Tabla 1.- Tasa de Actividad de Mujeres y Hombres en el año 1996 y en el año 2005 en Europa, España y Comunidad Valenciana. (Fuente: Eurostat, 2000 y 2005 y EPA 2000 y II Trimestre 2005)

\begin{tabular}{|l|l|l|l|l|}
\hline \multirow{2}{*}{} & \multicolumn{2}{|l|}{$\begin{array}{l}\text { TASA ACTIVIDAD MU- } \\
\text { JERES }\end{array}$} & $\begin{array}{l}\text { TASA ACTIVIDAD HOM- } \\
\text { BRES }\end{array}$ \\
\cline { 2 - 5 } \cline { 5 - 5 } & $\mathbf{1 9 9 6}$ & $\mathbf{2 0 0 5}$ & $\mathbf{1 9 9 6}$ & $\mathbf{2 0 0 5}$ \\
\hline UNIÓN EUROPEA & $57.5 \%$ & $62,1 \%$ & $78 \%$ & $77,5 \%$ \\
\hline ESPAÑA & $37.00 \%$ & $46,49 \%$ & $63.14 \%$ & $68,71 \%$ \\
\hline COM. VALENCIANA & $38.6 \%$ & $47,28 \%$ & $65.6 \%$ & $70,04 \%$ \\
\hline
\end{tabular}

Tabla 2.- Tasa de Paro de Hombres y Mujeres en el año 1996 y en el año 2005 en Europa, España y Comunidad Valenciana. (Fuente: Eurostat, 2000 y 2005 y EPA 2000 y II Trimestre 2005)

\begin{tabular}{|l|l|l|l|l|}
\hline \multirow{2}{*}{} & \multicolumn{2}{|l|}{ TASA PARO MUJERES } & \multicolumn{2}{l|}{ TASA PARO HOMBRES } \\
\cline { 2 - 5 } & $\mathbf{1 9 9 6}$ & $\mathbf{2 0 0 5}$ & $\mathbf{1 9 9 6}$ & $\mathbf{2 0 0 5}$ \\
\hline UNIÓN EUROPEA & $12.6 \%$ & $10 \%$ & $9.6 \%$ & $7,4 \%$ \\
\hline ESPAÑA & $29.56 \%$ & $12,22 \%$ & $17.60 \%$ & $7,29 \%$ \\
\hline COM. VALENCIANA & $30.0 \%$ & $12,03 \%$ & $16.6 \%$ & $7,31 \%$ \\
\hline
\end{tabular}

\section{Mujeres y Formación}

Los estudios realizados sobre los niveles, globales o sectoriales, de empleo de hombres y mujeres, han puesto de manifiesto la significatividad de los niveles de formación, no sólo en el acceso al empleo, sino también en la permanencia en el mismo. Comparativamente podemos apuntar que los hombres con poca formación tienen importantes niveles de desempleo, mostrando dificultades tanto en el acceso como en el mantenimiento de este, pero en los niveles de formación más elevados la dificultad más seria reside en el mantenimiento del empleo. Para las mujeres, las dificultades de inserción afectan a todos los niveles de formación, aunque con especial incidencia en los niveles más bajos, mientras que para los niveles más altos es destacable el aumento del paro de rotación (González Hernández y García Martínez, 1996).

Por otra parte, es importante analizar la concentración de las mujeres en unas pocas salidas profesionales, con escasas modificaciones en la evolución histórica. Como resul- tado de esta situación nos encontramos que los chicos están más orientados hacia formaciones más industriales y las chicas hacia formaciones del sector terciario (OIT, 1988). Esto podría ser denominado como "segregación sexual horizontal", que junto a la "segregación sexual vertical” referida a que las mujeres se formarían en profesiones con cualificaciones más bajas, conformarían el análisis de la evolución de las opciones de formación por sexos.

En cuanto a las cifras de las diferencias entre hombres y mujeres en cuanto a su formación, a nivel estatal, podemos apuntar que, la tasa de analfabetismo es del 4,22\% en el caso de las mujeres y de un $1.81 \%$ en el caso de los hombres, por otra parte en la educación infantil, la primaria y la secundaria la presencia de hombres y mujeres es prácticamente paritaria. En cuanto a los otros niveles formativos, universitarios y profesionales, que aportan una formación más focalizada hacia su posterior inserción laboral, paradójicamente tenemos un mayor número de mujeres (ver gráfico 1). 


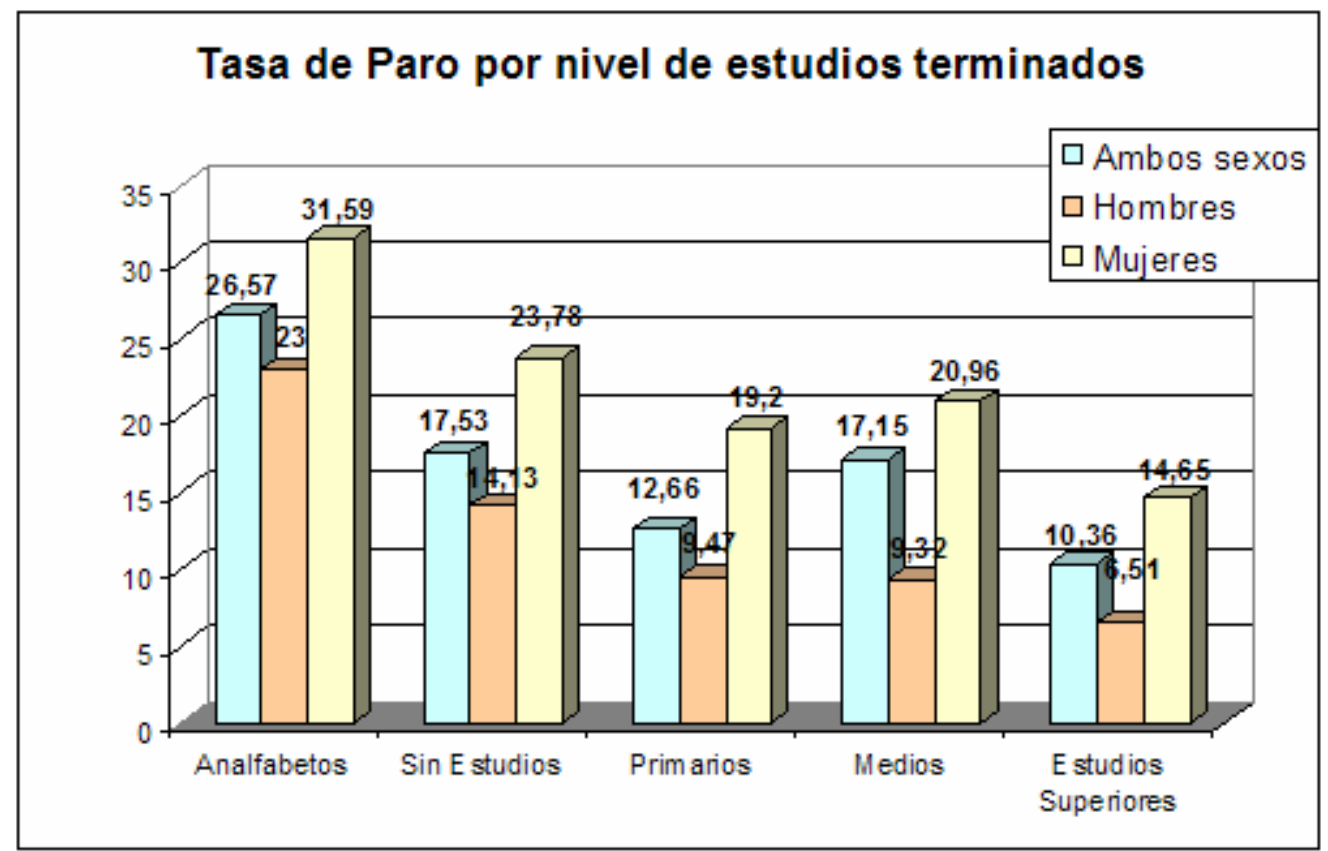

Gráfico 1.- Tasa de Paro por nivel de estudios terminados en ambos sexos, en hombres y en mujeres (Indicadores Sociales 2003: Fuente INE)

Sin embargo, la formación femenina sigue orientada hacia profesiones poco relevantes en el mercado de trabajo. Este parece un compromiso aceptable (Duru-Bellat, 1990) por parte de los sistemas democráticos, para evitar la puesta en cuestión del actual reparto del trabajo y poder entre los sexos, tanto en la vida profesional como hogareña.

Ante esta situación se confirma la persistencia de las desigualdades entre hombres y mujeres en la formación y se hace necesario potenciar además de una legislación sociolaboral favorable (cuidado y custodia de hijos, no gravamen de la maternidad para la mujer...), un proceso educativo y formativo que tenga en cuenta factores decisivos para potenciar la igualdad de oportunidades entre hombres y mujeres.

\section{Objetivos}

El Objetivo Principal de este artículo es: "Evaluar la calidad de los programas de Formación Ocupacional a partir de la opinión de las mujeres que participan en los mismos respecto al servicio que prestan y a su eficacia para lograr la inserción laboral de este colectivo".

A partir de este objetivo principal se podrían extraer tres objetivos operativos básicos:

- Analizar la satisfacción del cliente (mujeres que han recibido Formación Ocupacional) respecto a la formación recibida.

- Analizar la eficacia de los programas de Formación Ocupacional para las mujeres respecto a inserción laboral.

- Extraer posibles medidas de mejora para planificar los distintos programas de Formación Ocupacional y que, de este modo, atiendan de forma más adecuada las necesidades e intereses de este colectivo particular.

\section{Metodología}

El estudio que presentamos se define como un proceso de investigación evaluativa, dirigido a recabar información acerca de las acciones de Formación Ocupacional y la eficacia de éstas para conseguir la inserción laboral del colectivo de mujeres. Este proceso de investigación evaluativa estaría dentro de un 
proceso más extenso llamado Modelo EFO (Evaluación de la Formación Ocupacional), basado en una evaluación real, realizada en un contexto natural, lo que supone una de las mayores riquezas de la investigación evaluativa. Así, muchos de los factores de invalidez o de control de las interpretaciones que pueden controlarse en la investigación educativa, en este contexto deben de ser atendidos como indicios o evidencias, si se quiere más débiles pero extraídos de la situación real.

La investigación aquí planteada se ha estructurado en base a unos principios de funcionamiento que guían todos los análisis realizados: se centra en una aproximación basada en la complementariedad metodológica (cuantitativa/cualitativa) y dando importancia a los resultados descriptivos como base de reflexión. Además, se ha seguido en todo momento el planteamiento teórico sobre Metodología de Investigación Evaluativa que realizó Pérez Carbonell (1998).

Siguiendo la categorización de procesos de investigación presentada por Suárez (1989), desde el punto de vista metodológico se trata de: un estudio clásico observacional, de encuesta y de carácter diferencial.

\section{Presentación del Modelo EFO: marco metodológico del estudio presentado.}

En la Comunidad Valenciana (contexto en el cual se centra el estudio que aquí se presenta), a partir de 1993 se proponen introducir cambios en la gestión de la Formación Ocupacional, al objeto de mejorar su calidad. Estos cambios supusieron una reorganización de las Acciones Formativas, sobre todo respecto al sistema de adjudicación de subvenciones para el desarrollo de éstas, así como el control y seguimiento de su desarrollo. En este proceso de cambio forma parte fundamental la evaluación, ya que en la selección de solicitudes de las entidades colaboradoras se introdujo un doble sistema de evaluación, que combinaba la valoración técnica con la valoración emitida por un Comité de Expertos. Pero el papel de la evaluación no sólo se centraba en la selección de solicitudes, iba más allá, conllevaba la evaluación del desarrollo de los propios Programas de Formación Ocupacional, centrándose sobre todo en la Calidad de la Docencia de las Acciones Formativas. De este modo, se permitiría introducir innovaciones en la gestión y detectar puntos fuertes y débiles.

Este segundo aspecto de la evaluación, la Evaluación de Programas de Formación Ocupacional, fue encargada por la Consellería a un equipo del Departamento de Métodos de Investigación y Diagnóstico en Educación de la Universitat de Valencia, dirigido por los profesores Jesús M. Jornet y Jesús M. Suárez. En este proyecto se pretendía realizar el seguimiento y evaluación de todas las Acciones Formativas desarrolladas en el ejercicio 1996, creándose el MODELO EFO (Evaluación de la Formación Ocupacional). Finalizado este proyecto, en el año 2003 se requirió de nuevo la ayuda al equipo del Departamento MIDE por parte del SERVEF (organismo encargado de organizar la Formación Ocupacional y Continua) para analizar el sistema de evaluación de la Formación Ocupacional y Continua, así como diseñar su actualización.

El estudio que aquí se presenta, surge a partir del primer proyecto aludido en el párrafo anterior y sigue la línea de trabajo implementada por J.M. Jornet y J.M. Suárez (1996a, 1996b y 1996c) y continuada por M.J. Perales (2000, 2002).

Tomando como referencia base el Modelo de Evaluación de la Formación Ocupacional en la Comunidad Valenciana (Modelo EFO) (este modelo ya fue explicado en la Tesis de la cual surge este artículo Chiva, 2003 y también en otros trabajos relacionados Perales, 2000,2002), implementado en la convocatoria del ejercicio 1996 se pretende en este punto especificar cuáles son las características diferenciales y específicas que se van a utilizar para la Evaluación de Programas de 
Formación Ocupacional para el colectivo de mujeres.

\section{Primero, respecto al Objeto de evaluación} partiendo de este Modelo de Evaluación se intentará evaluar la calidad de la Docencia en las distintas acciones formativas del Programa de Formación Ocupacional, centrándonos tanto en el análisis de la Calidad del Servicio (satisfacción del colectivo de mujeres) como en la calidad del Programa (impacto de la acción formativa, integración sociolaboral). De este modo, la unidad de análisis principal $\mathrm{y}$, al mismo tiempo, fuente de información son las mujeres, comparado siempre con el colectivo de hombres.

Segundo, respecto a los Momentos de recogida de información en este estudio, se centran en datos recogidos de los alumnos/as respecto al Segundo Momento, una vez transcurrido el $80 \%$ de la acción formativa (es este pase el que realmente tiene un verdadero carácter evaluativo, pues se realiza cuando los alumnos/as tienen suficiente información para emitir un juicio informado, ya que en el Primer Momento, al 20\% de la acción formativa, cuentan con poca información) con el objeto de recoger la valoración respecto a la satisfacción en los Programas de Formación Ocupacional y del Tercer Momento, seis meses después de concluida la acción formativa, para recoger la eficacia respecto a inserción laboral de los participantes en el Programa de Formación Ocupacional.

Tercero, los Instrumentos utilizados concretamente para recoger información, son el Cuestionario de Opinión de Alumnos/as denominado EFO 2 (utilizado en el Segundo Momento) y el Cuestionario de Impacto (utilizado en el Tercer Momento).

\section{Muestras.}

Debido a que el artículo que aquí se presenta implica dos estudios distintos, estudio de la satisfacción de los clientes respecto a los programas formativos recibidos (EFO 2) y estudio del impacto de las acciones formativas para la inserción laboral (estudio de impacto) se han utilizado distintas muestras que a continuación presentamos someramente (ver tabla 3).

Las muestras en ambos estudios surgen de las valoraciones emitidas por los alumnos/as que habían participado en el Programa de Formación Ocupacional desarrollado por la Consellería de Trabajo y Asuntos Sociales de la Generalitat Valenciana en 1996. En el caso de la valoración de la satisfacción del cliente (EFO 2) se trata de un estudio poblacional ya que se recogen las valoraciones de todos los alumnos/as que participaron en cada una las acciones formativas realizadas durante ese año, de aquí que contemos con 16773 valoraciones emitidas a partir de cuestionarios pasados una vez transcurrido el $80 \%$ de la acción formativa. En el caso del estudio de impacto no se abarca a toda la población sino sólo una muestra por lo que tan sólo contamos con 1067 cuestionarios contestados de alumnos/as participantes en diversas acciones formativas. Además hemos dividido la muestra en función de la variable sexo ya que de esta manera podemos realizar un estudio diferencial de la valoración que realizan las mujeres del servicio recibido y de la inserción conseguida por las mismas.

\begin{tabular}{|l|c|c|}
\cline { 2 - 3 } \multicolumn{1}{c|}{} & $\begin{array}{c}\text { EFO 2 } \\
\mathbf{2}^{\mathbf{0}} \text { Pase }\end{array}$ & $\begin{array}{c}\text { Estudio } \\
\text { Impacto }\end{array}$ \\
\hline Total Alumnos/as & 16773 & 1067 \\
\hline Total Mujeres & 9909 & 583 \\
\hline Total Hombres & 6578 & 482 \\
\hline \multicolumn{2}{|c|}{ Tabla 3.- Muestra utilizada: número de valoraciones } \\
totales, de las mujeres y de los hombres
\end{tabular}

Estudiaremos las características de las muestras utilizadas específicamente cuando revisemos los resultados de cada uno de los estudios.

\section{6.- Resultados.}

A continuación se presentan los resultados obtenidos en las evaluaciones realizadas, comprobando: en primer lugar, la satisfac- 
ción de las mujeres respecto a las acciones formativas recibidas y, en segundo lugar, la eficacia de las acciones formativas para que este colectivo encuentre empleo, de esta manera se pretende responder a los objetivos operativos marcados en el estudio.

\section{1.- Resultados obtenidos respecto a la} satisfacción del colectivo de mujeres respecto a las acciones formativas recibidas.

En este apartado se trata de reflejar los resultados del estudio descriptivo de las valoraciones de las alumnas respecto a la acción formativa, una vez ha transcurrido el $80 \%$ de ésta (segundo pase), para conseguir así evaluar la calidad del servicio. Para llevar a cabo esta evaluación se ha utilizado el cuestionario denominado EFO 2 con 24 items. Estos ítems se estructuran en dimensiones establecidas a priori, por el consenso entre los distintos colectivos implicados en la evaluación. Las dimensiones son: Infraestructura, Organización, Profesorado, Metodología Docente, Adecuación de las Prácticas, Empleo y Valoración General.

En primer lugar, presentaremos la muestra concreta del estudio y a continuación los resultados obtenidos a partir de las valoraciones de los alumnos/as.

\section{Muestra}

Contamos con 16.773 valoraciones de alumnos/as que participaron en el Programa General de Formación Ocupacional denominado Programa Inserción al Trabajo, y de estas valoraciones 6.578 son valoraciones emitidas por mujeres. Estudiemos un poco más cuáles son las características que descri- ben a la muestra general y, concretamente, al colectivo de mujeres, con el objetivo de contextualizar y comprender los resultados que se obtienen.

Datos Personales: Sexo de los Participantes.

Un 60\% de los participantes en el Programa de Inserción al Trabajo son mujeres, por lo que se trataría del colectivo mayoritario.

\section{Datos Personales: Edad de los Participan-} tes.

El grupo de edad más frecuente tanto en hombres como en mujeres es el de 22-27 años, concretamente en este grupo de edad tenemos al $40 \%$ de las mujeres y al $35 \%$ de los hombres. El siguiente grupo de edad más frecuente es el de 16 a 21 años, donde aparecen un $28 \%$ de mujeres y un $34 \%$ de hombres. El resto de la muestra se distribuye en ambos grupos siguiendo una curva decreciente tal y conforme aumenta la edad. Como podemos deducir de esta distribución las mujeres empiezan más tarde a recibir este tipo de formación y también encontramos menor número de ellas en edades superiores a los 46 años.

Respecto a la edad media de los participantes, existen medias muy similares en hombres y mujeres cercanas al 2,2 (hay que tener en cuenta que la escala utilizada en esta variable es la siguiente: $1=16-21$ años, $2=22-27$ años, 3=28-33 años, 4=34-39 años, 5=40-45 años, 6=46-51 años y 7=52-57 años) Respecto a la forma de las distribuciones podrían calificarse de heterogéneas $(\mathrm{C} . \mathrm{V} .=$ superior al 50\%), demostrando una clara asimetría positiva y leptocurtosis. 


\begin{tabular}{|l|c|c|c|c|c|c|}
\hline \multicolumn{7}{|c|}{ Segundo Pase (EFO2) } \\
\hline \multirow{2}{*}{ Edad } & \multicolumn{3}{|c|}{ Mujeres } & \multicolumn{3}{c|}{ Hombres } \\
\cline { 2 - 7 } & Frecuencia & Porcentaje & \% válido & Frecuencia & Porcentaje & \% válido \\
\hline $16-21$ años & 2712 & 27.4 & 27.5 & 2195 & 33.4 & 33.6 \\
\hline $22-27$ años & 4319 & 43.6 & 43.9 & 2391 & 36.3 & 36.6 \\
\hline $28-33$ años & 1483 & 15.0 & 15.1 & 985 & 15.0 & 15.1 \\
\hline $34-39$ años & 753 & 7.6 & 7.6 & 413 & 6.3 & 6.3 \\
\hline $40-45$ años & 389 & 3.9 & 4.0 & 270 & 4.1 & 4.1 \\
\hline $46-51$ años & 141 & 1.4 & 1.4 & 157 & 2.4 & 2.4 \\
\hline $52-57$ años & 41 & .4 & .4 & 96 & 1.5 & 1.5 \\
\hline $58-65$ años & 8 & .1 & .1 & 27 & .4 & .4 \\
\hline No contesta & 63 & .6 & & 44 & .7 & - \\
\hline Total & 9909 & 100.0 & 100.0 & 6578 & 100.0 & 100.0 \\
\hline \multicolumn{7}{|c|}{ Tabla 4.- Distribución muestral de Mujeres y Hombres en el Programa Inserción al } \\
\hline
\end{tabular}

\section{Datos Académicos de los Participantes.}

Lo más frecuente entre los participantes del programa de Inserción al trabajo es que hayan cursado estudios medios (BUP O FP) (44\% están en esta situación, según el estudio de Perales, 2000). Esta misma tendencia se repite tanto en el grupo de las mujeres como en los hombres, aunque con distintas proporciones, más de un $47 \%$ de las mujeres están en esa situación académica, como se puede apreciar en la tabla, y tan sólo un $40 \%$ de los hombres.

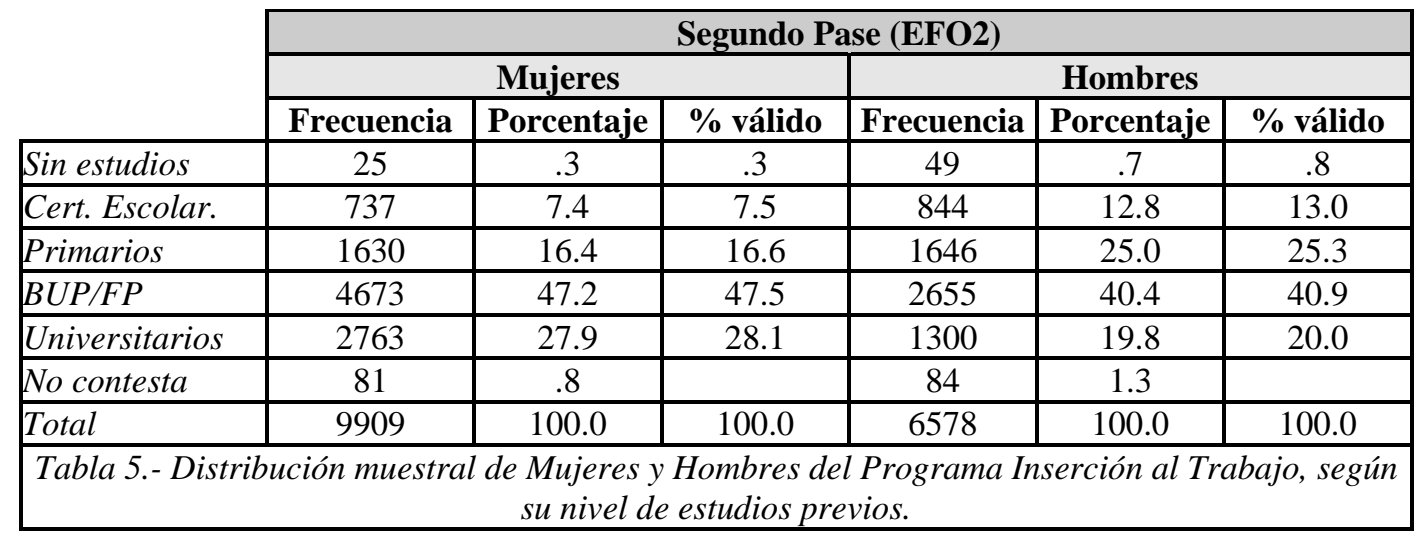

Especificando un poco más, en el caso de las mujeres entre las dos categorías de estudios medios y universitarios se recoge más de un $74 \%$, por lo que tan sólo un $26 \%$ tienen una formación inferior a los estudios medios, $\mathrm{y}$, en concreto sólo un $0.4 \%$ no tiene estudios previos. En el caso de los hombres esta distribución varia, ya que un $40 \%$ tiene estudios medios, un $19.8 \%$ tiene estudios universitarios, aunque hay un 38\% de hombres cuya formación no pasa de los estudios primarios. Estos datos nos indican que las mujeres tienen una mejor formación de salida que los hombres.

Como podemos observar el Programa Inserción al Trabajo tiene una oferta de acciones formativas que es capaz de atraer a personas con una formación diversa, con el propósito común de mejorar las opciones de encontrar empleo. Así, encontramos a participantes sin estudios y con estudios universitarios. 


\section{Datos Profesionales de los Participantes.}

Es obvio que el programa de Formación Profesional Ocupacional está específicamente dirigido a los desempleados/as, de modo que partimos de esta situación laboral en todos ellos.
Sin embargo, no es tan obvio el tiempo que llevan buscando empleo, de aquí la importancia del estudio de esta variable. Lo más frecuente es que lleven menos de un año buscando empleo, pero hay un pequeño grupo de personas que llevan más de seis años en esta situación (3,8\% de las mujeres y 2,2\% de los hombres) (ver Tabla 6).

\begin{tabular}{|c|c|c|c|c|c|c|}
\hline & \multicolumn{6}{|c|}{ Segundo Pase (EFO2) } \\
\hline & \multicolumn{3}{|c|}{ Mujeres } & \multicolumn{3}{|c|}{ Hombres } \\
\hline & Frecuencia & Porcentaje & \% válido & Frecuencia & Porcentaje & \% válido \\
\hline Sin estudios & 25 & .3 & .3 & 49 & .7 & .8 \\
\hline Cert. Escolar. & 737 & 7.4 & 7.5 & 844 & 12.8 & 13.0 \\
\hline Primarios & 1630 & 16.4 & 16.6 & 1646 & 25.0 & 25.3 \\
\hline$B U P / F P$ & 4673 & 47.2 & 47.5 & 2655 & 40.4 & 40.9 \\
\hline Universitarios & 2763 & 27.9 & 28.1 & 1300 & 19.8 & 20.0 \\
\hline No contesta & 81 & .8 & & 84 & 1.3 & \\
\hline Total & 9909 & 100.0 & 100.0 & 6578 & 100.0 & 100.0 \\
\hline
\end{tabular}

Si estudiamos la distribución de manera más pormenorizada comprobaremos que un elevado número de mujeres (41\%) llevan menos de un año buscando empleo, aunque cerca del $49 \%$ llevan de 1 a 4 años y cerca de un $10 \%$ lleva de 4 a más de 6 años. En el caso de los hombres está situación varía ya que un 52\% lleva menos de un año buscando empleo, cerca del $40 \%$ lleva entre 1 y 4 y tan sólo un 6\% lleva de 4 a más de 6 años. Estos datos nos indican varias cosas:

- Más del 70\% de los participantes en el programa de inserción al trabajo llevan como máximo dos años buscando empleo, lo que es coherente si tenemos en cuenta su edad (la mayoría son jóvenes) y su formación (la mayoría tienen estudios medios o superiores)

- Y la situación de la mujer en cuanto a tiempo de búsqueda de empleo es más precaria que en el caso de los hombres, ya que se indica que llevan más tiempo buscando empleo.

Dentro de los datos profesionales es importante estudiar si reciben o no la prestación por desempleo que es una de las medidas sociales desarrolladas bajo el estado del bienestar que reduce las repercusiones sociales del desempleo. Esa prestación disminuye las consecuencias negativas del tiempo de tránsito entre un empleo y otro, permitiendo contar con ciertos ingresos. Ante un mercado laboral tan cambiante, y con tanto flujo de personas, este tipo de medidas permite atenuar los cambios en las situaciones individuales (Perales, 2000).

La prestación por desempleo permite a la Administración intervenir en el proceso de búsqueda de empleo de las personas, intentando que sea más activo y eficaz. Los individuos pueden vivir ese "proteccionismo" de la Administración como la contrapartida de la prestación que están recibiendo.

En este contexto, el Programa de Formación Ocupacional puede ser una opción que realmente incremente las opciones de empleo de las personas y, por tanto, la Administración puede considerar que es una medida especialmente interesante para quienes están cobrando esa prestación. De hecho, en oca- 
siones la Administración ha propuesto a personas desempleadas con prestación la participación en Acciones Formativas que, por su trayectoria personal y laboral, podían ser especialmente adecuadas.

La participación obligatoria en esas Acciones Formativas se ha revelado como una medida ineficaz cuando se plantea como tal. Además, desde la gestión de las Acciones Formativas se han desarrollado mecanismos para evitarla, pues puede ser una influencia negativa para el propio curso. Probablemen- te, dentro de ese contexto de formación, una orientación integral permita adecuar las medidas a las personas, e incluso realizar un seguimiento de las personas, con lo que este tipo de medidas puedan ser reinterpretadas de un modo más eficaz.

El porcentaje de mujeres que recibe prestación por desempleo es de sólo un 14\%, frente a un $20 \%$ de los hombres (Tabla 7). Esto nos indica de nuevo que la mujer se encuentra en una situación más precaria que el hombre en el mercado laboral.

\begin{tabular}{|l|c|c|c|c|c|c|}
\cline { 2 - 7 } \multicolumn{1}{c|}{} & \multicolumn{6}{c|}{ Mujeres } \\
\cline { 2 - 7 } \multicolumn{1}{c|}{} & \multicolumn{5}{c|}{ Hegundo Pase (EFO2) } \\
\cline { 2 - 7 } & Frecuencia & Porcentaje & \% válido & Frecuencia & Porcentaje & \% válido \\
\hline No & 1356 & 13.7 & 13.8 & 1264 & 19.2 & 19.5 \\
\hline No contesta & 8460 & 85.4 & 86.2 & 5234 & 79.6 & 80.5 \\
\hline Total & 93 & .9 & & 80 & 1.2 & 100.0 \\
\hline Tabla 7.- Distribución muestral de Mujeres y Hombres del Programa Inserción al Trabajo, según \\
cobren o no prestación. \\
\hline
\end{tabular}

Datos relativos a la Acción Formativa que realizan.

A través del estudio de Perales (2000) comprobamos que la mayoría de los participantes en el programa de inserción asistían por primera vez a una acción formativa (84\% de la muestra).

Si diferenciamos entre hombres y mujeres observamos que no existen claras diferencias entre ambos grupos, ni tampoco respecto a la muestra general.

\begin{tabular}{|l|c|c|c|c|c|c|}
\cline { 2 - 7 } \multicolumn{1}{c|}{} & \multicolumn{6}{c|}{ Megundo Pase (Efo 2) } \\
\cline { 2 - 7 } \multicolumn{1}{c|}{} & \multicolumn{5}{c|}{ Mujeres } & \multicolumn{3}{c|}{ Hombres } \\
\cline { 2 - 7 } & Frecuencia & Porcentaje & \% válido & Frecuencia & Porcentaje & \% válido \\
\hline No & 1544 & 15.6 & 15.7 & 961 & 14.6 & 14.8 \\
\hline No contesta & 8288 & 83.6 & 84.3 & 5553 & 84.4 & 85.2 \\
\hline Total & 77 & .8 & & 64 & 1.0 & \\
\hline Tabla 8.- Distribución muestral de Mujeres y Hombres del Programa Inserción al Trabajo, \\
según hayan realizado o no otra acción formativa antes. \\
\hline
\end{tabular}

Otra variable importante dentro de los datos relativos al curso es descubrir si los participantes en los programas formativos tienen experiencia laboral en el área propia de cada acción formativa. En general, la mayoría no tienen experiencia laboral en el área (más del
$77 \%$ de la muestra). Aunque podemos observar que hay un tanto por cien más elevado de hombres (22-23\%) que de mujeres (18-19\%) que afirman tenerla (ver Tabla 9). Para este grupo de gente la acción formativa les permite actualizar conocimientos y tener el respal- 
Chiva, I. (2006). Evaluación de los programas de formación ocupacional para el colectivo de mujeres.

RELIEVE, v. 12, n. 1, p. 49-74. http://www.uv.es/RELIEVE/v12n1/RELIEVEv12n1_3.htm

do de un título, para continuar buscando em- $\quad$ pleo en el mismo sector profesional.

\begin{tabular}{|l|c|c|c|c|c|c|}
\cline { 2 - 7 } \multicolumn{1}{c|}{} & \multicolumn{6}{c|}{ Segundo Pase (Efo 2) } \\
\cline { 2 - 7 } \multicolumn{1}{c|}{} & \multicolumn{4}{c|}{ Mujeres } & \multicolumn{3}{c|}{ Hombres } \\
\cline { 2 - 7 } & Frecuencia & Porcentaje & \% válido & Frecuencia & Porcentaje & \% válido \\
\hline Si & 1946 & 19.6 & 19.9 & 1505 & 22.9 & 23.1 \\
\hline No & 7857 & 79.3 & 80.1 & 4997 & 76.0 & 76.9 \\
\hline To contesta & 106 & 1.1 & & 76 & 1.2 & \\
\hline
\end{tabular}

Tabla 9.- Distribución muestral de Mujeres y Hombres del Programa Inserción al Trabajo, según tengan o no experiencia laboral en el sector de la A. F. que cursan.

Datos relativos a las familias profesionales de la acción formativa que realizan.
Concretamente, la distribución de hombres y mujeres en cuanto a las distintas familias profesionales muestra diferencias entre ambos grupos (Tabla 10).

\begin{tabular}{|l|c|c|}
\cline { 2 - 3 } \multicolumn{1}{c|}{} & \multicolumn{2}{c|}{ Segundo pase (Efo 2) } \\
\cline { 2 - 3 } \multicolumn{1}{c|}{} & \% Mujeres & \%Hombres \\
\hline Agraria & 1.7 & 5.4 \\
\hline Administr y Oficinas & 32.3 & 13.8 \\
\hline Artesanía & 0.9 & 0.2 \\
\hline Automoción & .0 & 5.0 \\
\hline Comercio & 13.8 & 7.4 \\
\hline Docencia e Investig. & 2.9 & 1.9 \\
\hline Serv a las Empresas & 20.6 & 17.8 \\
\hline Edificación y Obras & 0.9 & 9.4 \\
\hline Ind. Equipos mecánic & 0.3 & 3.6 \\
\hline Ind. Alimentarias & 0.8 & 1.1 \\
\hline Ind. Gráficas & 0.6 & 1.0 \\
\hline Inf. y manif. artíticas & 0.5 & 1.3 \\
\hline Ind. Pesada & 0.0 & 1.4 \\
\hline Ind. Química & 0.1 & 0.6 \\
\hline Ind. Textiles & 4.0 & 2.2 \\
\hline Ind. Madera y corcho & 0.3 & 2.4 \\
\hline Mantenim y reparac. & 0.2 & 6.5 \\
\hline Montaje e instalación & 0.4 & 9.7 \\
\hline Pesca y acuicultura & 0.1 & 0.1 \\
\hline Prod. Energía & - & 0.2 \\
\hline Sanidad & 6.1 & 0.7 \\
\hline Serv. Comunidad & 8.7 & 2.9 \\
\hline Transp y Comunicac & 0.3 & 100 \\
\hline Turismo y Hostelería & 4.3 & \\
\hline Total & $100 \%$ & Mombres del \\
\hline Tabla 10. Distribución muestral de Mujeres y & \\
\hline Programa Inserción al Trabajo, según las familias profesio- \\
\hline \multicolumn{1}{|c|}{ nales del curso que realizan. } & \\
\hline & & \\
\hline
\end{tabular}

Más pormenorizadamente podemos decir que existen claramente dos tipos de acciones formativas: las eminentemente femeninas $\mathrm{y}$ las eminentemente masculinas, a continuación se ofrece una tabla donde aparecen las 
familias más representativas de cada uno de los grupos:

\begin{tabular}{|l|l|}
\hline $\begin{array}{l}\text { Familias Profesionales con } \\
\text { mayor porcentaje femenino }\end{array}$ & $\begin{array}{l}\text { Familias Profesionales con } \\
\text { mayor porcentaje masculino }\end{array}$ \\
\hline Administración y Oficinas & Servicios a las Empresas \\
\hline Servicios a las Empresas & Administración y Oficinas \\
\hline Comercio & Montaje e Instalación \\
\hline Servicios a la Comunidad & Edificación y Obras \\
\hline Sanidad & Comercio \\
\hline $\begin{array}{l}\text { Tabla 11.- Distribución de familias profesionales en función de si } \\
\text { tienen mayor porcentaje de Mujeres o de Hombres en el Programa } \\
\text { Inserción al Trabajo. }\end{array}$ \\
\hline
\end{tabular}

Si analizamos los tipos de familias profesionales en función de la variable Sexo, podemos observar que se refleja un poco el estereotipo social existente entre hombres y mujeres, ya que se asume que las mujeres están más capacitadas para profesiones relacionadas con la administración y oficinas, servicios a las empresas, comercio, servicios a la comunidad... mientras que los hombres son mayoritarios en familias profesionales más relacionadas con el trabajo físico, como: montaje e instalación, edificación, ...

Tampoco hay que olvidar que la situación está empezando a cambiar, ya que se observa en el porcentaje de hombres que la familia profesional más frecuente es servicios a las empresas, administración y oficinas (aunque son las mujeres las que tienen mayor porcentaje en ambas). Y por otro lado, la mujer está ampliando más sus perspectivas formativas incluyéndose en campos como agraria, edificación,... que en otros tiempos hubiera sido imposible ver a una sola mujer en este tipo de curso.

Por último, anotar que las mujeres se concentran en tres familias profesionales bási- camente, esto supone que el $66 \%$ de la muestra se sitúa en familias como administración y oficinas, servicios a las empresas y comercio, mientras que los hombres se diversifican más ya que en cuatro familias profesionales se concentran sólo el $50 \%$ de la muestra (servicios a las empresas, administración y oficinas, montaje e instalación y edificación y obras)

\section{Resultados}

Mediante este punto se pretende ofrecer una visión general descriptiva de las valoraciones de los alumnos/as en el Segundo Pase (EFO 2) respecto a su satisfacción con el Programa Inserción al Trabajo de Formación Ocupacional, distinguiendo entre las valoraciones de los hombres y las mujeres para comparar y observar si existen diferencias en cuanto a la satisfacción de estos dos colectivos.

En las tablas siguientes se presentaran las valoraciones de los participantes en los programas de inserción al trabajo en cada uno de los ítems y a continuación en cada una de las dimensiones que engloban las distintas temáticas que se abordan en el cuestionario. 


\begin{tabular}{|c|c|c|c|c|}
\hline \multirow{2}{*}{$\begin{array}{l}\text { INSERCIÓN AL TRABAJO } \\
\text { Segundo Pase }\end{array}$} & \multicolumn{2}{|c|}{ Total Mujeres } & \multicolumn{2}{|c|}{ Total Hombres } \\
\hline & \multicolumn{2}{|c|}{ Med D.T. } & \multicolumn{2}{|c|}{ Med D.T. } \\
\hline 1. Se han dado todas las clases anunciadas ... & 4.55 & 4.44 & 4.44 & 0.82 \\
\hline 2. Se ha seguido el horario establecido ... & 4.46 & 4.40 & 4.40 & 0.92 \\
\hline 3. La Organización general del curso ... & 4.11 & 4.03 & 4.03 & 1.00 \\
\hline 4. El tiempo dedicado a las clases prácticas ... & 3.96 & 3.94 & 3.94 & 1.14 \\
\hline 5. La Infraestructura, equipos y materiales ... & 3.94 & 3.86 & 3.86 & 1.12 \\
\hline 6. Los materiales para las clases teóricas ... & 4.21 & 4.10 & 4.10 & 0.98 \\
\hline 7. Se han facilitado materiales de consumo ... & 4.19 & 4.10 & 4.10 & 1.07 \\
\hline 8. Las explicaciones de los profesores/as... & 4.43 & 4.38 & 4.38 & 0.82 \\
\hline 9. Los profesores han sido respetuosos ... & 4.67 & 4.65 & 4.65 & 0.69 \\
\hline 10. Los profesores utilizan diversos medios ... & 4.31 & 4.23 & 4.23 & 0.94 \\
\hline 11. La comunicación entre profesor/alumno/a ... & 4.60 & 4.56 & 4.56 & 0.72 \\
\hline 12. El profesor intenta que participemos ... & 4.59 & 4.51 & 4.51 & 0.71 \\
\hline 13. Mi trabajo se ha valorado diariamente ... & 3.83 & 3.79 & 3.79 & 1.10 \\
\hline 14. Hemos hecho prácticas con seguridad ... & 4.23 & 4.20 & 4.20 & 0.98 \\
\hline 15. Las prácticas tienen que ver con el trabajo real.. & 4.01 & 3.97 & 3.97 & 1.03 \\
\hline 16. Las prácticas están relacionadas con la teoría. & 4.34 & 4.27 & 4.27 & 0.89 \\
\hline 17. Este Centro ha realizado gestiones ... empleo. & 3.16 & 3.11 & 3.11 & 1.23 \\
\hline 18. Han estudiado mis cualidades para ... empleo & 3.23 & 3.23 & 3.23 & 1.27 \\
\hline 19. Se nos ha informado sobre ... buscar empleo & 3.54 & 3.43 & 3.43 & 1.22 \\
\hline 20. Este curso ha ampliado mi campo profesional .. & 4.18 & 4.18 & 4.18 & 0.90 \\
\hline 21. ... me siento capacitado para esta ocupación. & 3.93 & 3.91 & 3.91 & 0.99 \\
\hline 22. Han aumentado mis posibilidades ... empleo & 4.01 & 3.99 & 3.99 & 0.95 \\
\hline 23. En general, este curso es de buena calidad. & 4.20 & 4.10 & 4.10 & 0.92 \\
\hline 24. Recomendaría a otras personas ... este curso & 4.33 & 4.20 & 4.20 & 0.95 \\
\hline 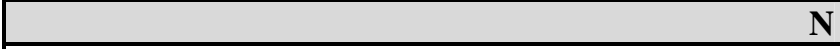 & \multicolumn{2}{|c|}{9909} & \multicolumn{2}{|c|}{6574} \\
\hline
\end{tabular}

En primer lugar, comentar que las valoraciones de ambos grupos, hombres y mujeres, son bastante positivas, ya que superan en casi todos los items el punto 4 de la escala, hay que tener en cuenta que los ítems están diseñados con una escala Likert, con cinco opciones de respuesta: totalmente en desacuerdo (1), bastante en desacuerdo (2), indeciso (3), bastante de acuerdo (4) y totalmente de acuerdo (5).

En cuanto a la valoración media de los hombres estamos hablando de un 4.06 y un 4,12 en el caso de las mujeres. Estos valores se corresponden con la idea de que las mujeres valoran más alto, y por tanto se muestran más satisfechas con las acciones formativas que los hombres.

Es importante destacar que al igual que en el Primer Pase aparecen algunos items que no alcanzan este nivel medio ni llegan a la puntuación 4 y que por tanto podríamos denominar como los más críticos:

- Ítem 4 "El tiempo dedicado a las clases prácticas ..."

- Ítem 5 "La Infraestructura, equipos y materiales..."

- Ítem 13 "Mi trabajo se ha valorado diariamente..."

- Ítem 17 "Este centro ha realizado gestiones... empleo"

- Ítem 18 "Han estudiado mis cualidades para ... empleo"

- Ítem 19 "Se nos ha informado sobre... buscar empleo"

- Ítem 21 “... me siento capacitado para esta ocupación”

De manera general, las valoraciones emitidas por los participantes al $80 \%$ de realizada la acción formativa se podrían sintetizar en que se da una valoración global positiva (4.06 en hombres y 4.12 en mujeres sobre 5 
puntos), un poco inferior a la emitida al 20\%:4.23 en hombres y 4.32 en mujeres (los resultados respecto a las valoraciones emitidas al $20 \%$ de la acción formativa aparecen en la tesis doctoral de Chiva, 2003), y en la que hay mayores matices, pues aumentan el número de items que no llegan a la puntuación 4, se introduce mayor variabilidad y hay menor concentración de puntuaciones. Esta variación sucede puesto que los alumnos/as tienen mayores elementos de juicio mostrándose más críticos, y tratan de reflejar las distintas situaciones vividas por lo que se incrementa la variabilidad en las puntuaciones.

Por otra parte, si realizamos una comparación entre los grupos de hombres y mujeres (mediante el correspondiente análisis de varianza) comprobaremos que existen diferencias significativas (los resultados derivados del análisis de varianza, donde se observan las diferencias significativas anunciadas entre ambos colectivos están especificados en Chiva, 2003) en las valoraciones de los participantes en las distintas acciones formativas, en función de ser hombre o mujer debido a que las mujeres suelen valorar más positivamente que los hombres. Esto supone que todos los items tienen valoraciones con dife- rencias significativas en función de ser hombre o mujer, a excepción de los items $4,9,13,14,18,20,21$ y 22 donde las valoraciones son más similares entre ambos grupos. Estos items, que no tienen diferencias significativas entre hombres y mujeres, coinciden, en su mayoría, con los denominados más críticos que no alcanzan la valoración de 4 y que se refieren básicamente a cuestiones sobre empleo.

Respecto a las dimensiones del cuestionario del Segundo Pase, en primer lugar hay que tener en cuenta que son valoradas de modo más crítico por los alumnos/as si las comparamos con las valoraciones de las dimensiones realizadas al $20 \%$ de la acción formativa, tanto en hombres como en mujeres, en segundo lugar apuntar que como muestra la tabla 13 existen diferencias significativas en todas las dimensiones evaluadas ya que de nuevo son las mujeres las que valoran más alto que los hombres en todas las dimensiones, y en tercer lugar las dimensiones peor valoradas son empleo, seguida por Infraestructura y la más valorada ha sido Profesorado, tanto en hombres como en mujeres, (ver Gráfico 2).

Gráfico 2.- Valoraciones de Mujeres y Hombres en las distintas dimensiones de Segundo pase.

\begin{tabular}{|l|c|c|c|c|c|c|c|}
\hline Dimensiones & Tot & $\mathbf{H}$ & $\mathbf{M}$ & $\begin{array}{c}\text { Sig. dife- } \\
\text { rencia }\end{array}$ & Valor sig. & $\begin{array}{c}\text { Homog. } \\
\text { varianzas }\end{array}$ & Tendencia \\
\hline Infraestructura & 4.07 & 4.01 & 4.10 & Si sig. & 0.001 & Si sig. & Menor satisfacción Hombres \\
\hline Organización & 4.23 & 4.20 & 4.26 & Si sig. & 0.001 & Si sig. & Menor satisfacción Hombres \\
\hline Profesorado & 4.53 & 4.51 & 4.54 & Si sig. & 0.003 & No sig. & Menor satisfacción Hombres \\
\hline $\begin{array}{l}\text { Metodología } \\
\text { Docente }\end{array}$ & 4.30 & 4.27 & 4.33 & Si sig. & 0.001 & Si sig. & Menor satisfacción Hombres \\
\hline $\begin{array}{l}\text { Adecuación de las } \\
\text { Prácticas }\end{array}$ & 4.14 & 4.11 & 4.16 & Si sig. & 0.000 & No sig. & Menor satisfacción Hombres \\
\hline Empleo & 3.67 & 3.65 & 3.68 & Si sig. & 0.013 & No sig. & Menor satisfacción Hombres \\
\hline $\begin{array}{l}\text { Valoración Gene- } \\
\text { ral }\end{array}$ & 4.21 & 4.14 & 4.26 & Si sig. & 0.001 & Si sig. & Menor satisfacción Hombres \\
\hline
\end{tabular}

Tabla 13. Resumen de Resultados del Análisis de Varianza para los grupos establecidos en función de la variable Sexo en las dimensiones del Programa Inserción al Trabajo en el Segundo Pase. 


\section{2.- Resultados obtenidos respecto al impacto de las acciones formativas en la inserción laboral del colectivo.}

El análisis del impacto de la Formación Ocupacional, concretamente en el programa de la Comunidad Valenciana, constituye una aproximación para la evaluación de la eficacia del mismo desde el punto de vista sociopolítico. Desde esta perspectiva, el grado de inserción profesional consecuente a la realización de una acción formativa se constituye en el criterio clave de referencia.

Aunque el nivel de inserción no es el único indicador de calidad sociopolítica del Programa de Formación Ocupacional ya que también es importante la valoración de la calidad del empleo conseguido, la duración..., si que es el más habitual y una referencia clave.

Por otro lado, la inserción laboral de los alumnos/as, por ahora, si bien es la finalidad última que justifica la realización del Programa de Formación Ocupacional no es asumida como tal por todos los implicados (Perales, 2000). Esto nos conduce a que el estudio se vea afectado por factores como: la escasez de personas insertadas y la disparidad de actuaciones a este respecto de las distintas entidades formativas que participan en el programa formativo.

No obstante, vamos a intentar evaluar el grado de inserción profesional, medida a medio plazo (seis meses después de haber concluido las acciones formativas) como un indicador de eficacia del Programa de Formación Ocupacional, concretamente para el colectivo de mujeres.

Para ello, en primer lugar presentaremos la muestra y el instrumento utilizado y a continuación los resultados obtenidos.

\section{Muestra}

La selección de la muestra se basó en un muestreo aleatorio de las acciones formativas realizadas en la convocatoria del programa de Formación Ocupacional en el año 1996. Para la selección se tuvo en cuenta: que la muestra fuera representativa a nivel del total de acciones formativas y que se respetara la estratificación por provincias de la Comunidad Valenciana y por Familia Profesional (Perales, 2000 y 2002). De este modo, se seleccionaron un total de 106 acciones formativas de las 1492 que habían sido evaluadas en segundo pase. Hay que tener en cuenta que no se pudo acceder a la totalidad de participantes que recibieron las acciones formativas seleccionadas y al final se contó con los datos de un total de 1067.

Centrándonos en el colectivo de mujeres, en este estudio se recogió la información de 583 mujeres, lo que supone un $55 \%$ de la muestra utilizada en el estudio del impacto. De este colectivo de mujeres podemos decir que:

- Existe bastante dispersión respecto a la edad, encontrando mujeres desde 16 a 60 años, aunque el mayor número aparece en edades comprendidas entre 20 y 26 años (cerca del 50\%), si comparamos con los hombres su edad más frecuente se encuentra entre los 17 y 24 años, diferencia que también se refleja en la media de edad: 27,29 en el caso de las mujeres y 25,68 en el caso de los hombres.

- El nivel de estudios más frecuente en las mujeres son los estudios de Bachillerato o Formación Profesional (49\%), al igual que sucede en los hombres (42\%) pero en menor medida, pues tenemos casí un 35\% de los hombres con estudios primarios.

- Al igual que ocurría en la descripción de la muestra del anterior estudio, de nuevo aparecen las mujeres con mayor edad y con mayor nivel de estudios cuando las comparamos con los hombres. 


\section{Instrumento}

La evaluación del impacto se realizó a través de la aplicación de una encuesta telefónica a los alumnos/as de la muestra. En esta encuesta, además de recoger una serie de datos personales y datos de la acción formativa, se preguntaba al alumno/a si tenía empleo, si había realizado otro curso de Formación Ocupacional y se le pedía una valoración del curso realizado de manera diferida.

\section{Resultados}

El primer indicador que podemos extraer es el nivel de inserción de las mujeres a los seis meses de haber finalizado la acción formativa, resultando que sólo un $20 \%$ del colectivo seleccionado habían conseguido un empleo, frente al $80 \%$ que continuaba en situación de desempleo (ver Tabla 14). Si comparamos con las cifras de los hombres, un $31 \%$ consiguieron empleo, por lo que podemos afirmar que el indicador es un poco más desfavorable para el colectivo de mujeres.

\begin{tabular}{|l|c|c|c|c|c|c|}
\cline { 2 - 7 } \multicolumn{1}{c|}{} & \multicolumn{6}{c|}{ Estudio Impacto } \\
\cline { 2 - 7 } \multicolumn{1}{c|}{} & \multicolumn{5}{c|}{ Mujeres } & \multicolumn{3}{c|}{ Hombres } \\
\cline { 2 - 7 } \multicolumn{1}{c|}{ Frecuencia } & Porcentaje & \% válido & Frecuencia & Porcentaje & \% válido \\
\hline No & 464 & 79,6 & 79,6 & 334 & 69,3 & 69,3 \\
\hline Total & 119 & 20,4 & 20,4 & 148 & 30,7 & 30,7 \\
\hline Tabla 14.-. Distribución de Mujeres y Hombres según tengan o no tengan empleo a los seis meses \\
de terminar el programa de Formación Ocupacional \\
\hline
\end{tabular}

De este $20 \%$ de mujeres que han consequido empleo, la mayoría superaban los $10 \mathrm{me}-$ ses en paro (55\%) y habían encontrado el empleo en menos de dos meses después de terminado el curso (más del 70\%). Respecto a si el empleo encontrado era de la misma especialidad del curso, el 62\% afirma que no estaba relacionado y en la mayoría de los casos se trataba de un trabajo temporal. Por último, tan sólo un 15\% encontraron empleo a través de la entidad formativa que impartía el curso. Si comparamos estos datos con lo que ocurre en el caso del colectivo de hombres podemos afirmar que existen diferencias respecto al tiempo en paro antes de encontrar el empleo ya que es menor que en las mujeres (al menos dos o tres meses) y respecto a si la entidad formativa es la que facilita el empleo ya que sólo se benefician de esta intervención un 5,6\% de los hombres, las demás respuestas son muy similares, también se trata de un trabajo temporal y con poca relación con el curso realizado.

Además de la información respecto al impacto de la acción formativa, analizada anteriormente, se preguntaba al encuestado/a si había realizado algún otro curso de Formación Ocupacional a lo que cerca del 68\% de las mujeres contestaba que no, por lo que se trataría de su primer contacto con la Formación Ocupacional (¿Quizás el último?), al igual que ocurre en el caso de los hombres (cerca del $70 \%$ afirman que es su primer contacto con la formación ocupacional). Para finalizar la encuesta telefónica, se pretendía recoger una valoración diferida del curso demostrando que a los seis meses de haber finalizado la acción formativa se sigue manteniendo una opinión muy positiva acerca del curso, más del 91\% de las mujeres entrevistadas creen que el curso fue bueno o muy bueno (ver Tabla 15), una opinión un poco más positiva que en el caso de los hombres. 


\begin{tabular}{|c|c|c|c|c|c|c|}
\hline & \multicolumn{6}{|c|}{ Estudio Impacto } \\
\hline & \multicolumn{3}{|c|}{ Mujeres } & \multicolumn{3}{|c|}{ Hombres } \\
\hline & Frecuencia & \begin{tabular}{|l|} 
Porcentaje \\
\end{tabular} & \% válido & Frecuencia & Porcentaje & \% válido \\
\hline Muy Malo & \begin{tabular}{|c|}
4 \\
\end{tabular} & ,7 & ,7 & 6 & 1,2 & 1,3 \\
\hline Malo & 15 & 2,6 & 2,6 & 26 & 5,4 & 5,4 \\
\hline Regular & 30 & 5,1 & 5,2 & 18 & 3,7 & 3,8 \\
\hline Bueno & 266 & 45,6 & 46,1 & 290 & 60,2 & 60,4 \\
\hline Muy bueno & 262 & 44,9 & 45,4 & 140 & 29,0 & 29,2 \\
\hline No contestan & 6 & 1,0 & ,7 & 2 & ,4 & 1,3 \\
\hline Total & 583 & 100,0 & 100,0 & 482 & 100,0 & 100,0 \\
\hline
\end{tabular}

\section{7.- Conclusiones y Comentarios Fina- les}

Antes de empezar con las conclusiones, debemos recoger de nuevo el objetivo principal del cual hemos partido "Evaluar la calidad de los programas de Formación Ocupacional a partir de la opinión de las mujeres que participan en los mismos respecto al servicio que prestan y a su eficacia para lograr la inserción laboral de este colectivo", con la finalidad de comprobar si lo hemos cumplido y a qué conclusiones hemos llegado.

De acuerdo con esta idea, vamos a empezar a reflexionar acerca del primero de los tres objetivos operativos que concretan al objetivo principal: "Analizar la satisfacción del cliente (en este caso las mujeres) respecto a la formación recibida" del cual podemos extraer las siguientes conclusiones:

- En primer lugar, podemos decir que las características de las mujeres que participan en Programa Inserción al Trabajo son las siguientes:

- Las mujeres empiezan a participar en las acciones formativas con más edad que los hombres, aunque hay menor número de ellas en edades superiores a 46 años.

- Las mujeres tienen mayor nivel de estudios que los hombres, ya que hay mayor porcentaje de ellas en estudios medios y universitarios.

- Las mujeres llevan más tiempo buscando empleo que los hombres y hay menor nú- mero de ellas que están recibiendo una prestación por desempleo.

- Las mujeres tienen menos experiencia laboral relacionada con el curso que están realizando.

- Las mujeres son mayoritarias en familias profesionales como: administración y oficinas, servicios a las empresas, comercio..., mientras que siguen estando en minoría en familias profesionales como montaje e instalación, edificación y obras...

- Caracterizado el colectivo de mujeres, hemos recogido las siguientes evidencias derivadas de los resultados obtenidos de las valoraciones de este colectivo respecto de las acciones formativas de Formación Ocupacional en las que participaron, transcurrido el $80 \%$ de las sesiones:

- Las mujeres están más satisfechas con la formación recibida en todos los ítems y dimensiones analizadas que los hombres.

- La dimensión mejor valorada es profesorado, seguida de Metodología docente, por lo que el punto fuerte de la calidad de la formación profesional ocupacional es el estilo docente.

- La dimensión peor valorada es empleo tanto para hombres como mujeres por lo que sería necesario mejorar estos aspectos que son valorados tanto por hombres como por mujeres menos satisfactoriamente.

- Por último, hemos analizado si existen diferencias entre hombres y mujeres: constatando que son significativas en casi todos los ítems, en todas las dimensiones y en los resultados globales de las valora- 
ciones debido a que las mujeres tienen unas valoraciones más positivas

Respecto al segundo objetivo "Analizar la eficacia de los programas de Formación Ocupacional para las mujeres respecto a inserción laboral" hemos extraído las siguientes conclusiones:

- En cuanto a la muestra de 1067 encuestados telefónicamente, un 55\% son mujeres (en total 583). Las características de la muestra son similares a las nombradas en las conclusiones del anterior objetivo: mayor nivel de estudios y con mayor edad si las comparamos con las características que presentan los hombres.

- Las evidencias derivadas de este estudio apuntan que:

- Tan sólo un 20\% de los mujeres encuestadas consiguieron empleo después de realizada la acción formativa, frente a un 31\% de los hombres. Empleo no relacionado con la acción formativa que habían recibido, empleo que no ha sido gestionado por la propia entidad formativa y empleo en la mayoría de casos temporal. Podemos afirmar que es bastante decepcionante que tan pocas mujeres hayan conseguido un empleo, cuando es realmente éste el objetivo básico de la Formación Ocupacional

- Las mujeres que consiguieron un empleo afirman llevar más de 10 meses en paro. Comparado con los hombres se constata que el número de meses en paro es mayor. - Generalmente, no han realizado otro curso de Formación Ocupacional tanto los hombres como las mujeres.

- Y por último, la valoración diferida acerca de la acción formativa realizada es muy positiva, un poco más positiva en las mujeres que en los hombres.

Respecto al tercer objetivo "Extraer posibles medidas de mejora para planificar los distintos programas de Formación Ocupacional y que de este modo atiendan de forma más adecuada las necesidades e intereses de este colectivo particular" se puede concluir que Aunque la satisfacción de las mujeres es muy alta respecto a las acciones formativas recibidas, hay aspectos a mejorar:

- Las Gestiones para el Empleo, sería necesario que fueran reforzadas por las entidades formativas ya que ha sido el apartado más criticado y sería una de las claves del éxito: lograr la inserción. Las entidades formativas deberían de asumir la inserción profesional de sus alumnas como una finalidad a conseguir, por lo que sería conveniente requerir un compromiso de inserción, así como mayores gestiones para lograr que encuentren un empleo, ya que como hemos visto (sólo un $20 \%$ de los mujeres consiguen un empleo) no se asume como un aspecto asociado a la acción formativa.

- El Profesorado, aunque es el aspecto mejor valorado de las acciones formativas, es el elemento más débil del sistema (generalmente son profesionales en paro, en situación de inestabilidad laboral...) de modo que la calidad docente que perciben los alumnos/as se debe al esfuerzo de personas aisladas. Sería necesario, por tanto, mejorar la situación de este colectivo para que su esfuerzo revierta en una mejora de la calidad de la Formación Ocupacional.

- Sería conveniente completar los Programas de Formación Ocupacional con actuaciones de Orientación laboral, de manera que se intervenga para facilitar el empleo, ofreciendo información y orientación adecuada a cada una de las participantes en las acciones formativas para aportar por un lado información necesaria sobre la construcción de su propio curriculum profesional y, por otro, para acercar las propuestas formativas a las demandas de las empresas y a las expectativas de las propias trabajadoras.

- Se necesitarían realizar cursos específicos para las mujeres, ya que las características de las cuales parten respecto a los hombres son distintas, lo que puede suponer una Mayor inversión económica.

- Es necesario que tanto a nivel europeo, estatal como en la Comunidad Valenciana 
se siga considerando a las mujeres como colectivo con riesgo de exclusión social y, como tal, que forme parte de las distintas políticas, acciones, programas... de empleo y formación establecidos y financiados con fondos públicos para: por un lado, vencer las dificultades estructurales del empleo y, por otro, formar para el trabajo de una manera más adecuada, eficaz y diferencialmente a las mujeres.

- Por último, vistos los resultados derivados de los tres objetivos operativos es importante plantearse la necesidad de seguir revisando y realizando un seguimiento continuo de las distintas acciones formativas de formación ocupacional en las que participa la mujer tanto respecto a la calidad del servicio que prestan como respecto a la eficacia de las mismas para la inserción laboral de este colectivo. Esta idea puede ser viable ya que todavía continua vigente un sistema similar de seguimiento y control del desarrollo de las distintas acciones formativas a nivel de Comunidad Valenciana. Por tanto, la evaluación de la Formación Ocupacional, y muy especialmente en el caso de colectivos desfavorecidos como son las mujeres, se convierte en un medio para comprobar si las acciones formativas son eficaces y eficientes para los colectivos a los que va dirigido y para los objetivos a los que pretenden responder.

Para finalizar con este artículo, comentar que se han producido, a nivel comunitario, estatal y autonómico, algunos progresos en los últimos años, pues se han puesto en marcha múltiples planes y programas para potenciar la igualdad de oportunidades en todos los sentidos, que están aportando resultados satisfactorios.

Sin embargo, la incorporación de las mujeres al mundo laboral y formativo no ha sido plena en la misma medida que los hombres, tal como nos muestran los datos aportados, ya que todavía sus contratos son los más deficitarios, su salario es menor por un trabajo igual, su presencia en los puestos de deci- sión es escasa, su dedicación al trabajo doméstico y familiar es superior a la de los hombres, y su formación está relacionada con unas pocas salidas profesionales (formaciones del sector terciario y de baja cualificación).

Podemos decir, pues, que sigue siendo necesario potenciar la igualdad de oportunidades entre hombres y mujeres a nivel social, cultural, educativo y laboral. En este mismo sentido y, centrándonos un poco en el tema de nuestro trabajo, si lo que queremos conseguir es una inserción laboral al mismo nivel de los hombres se deben potenciar las diversas actuaciones que se han ido apuntando como: acceso a los derechos sociales y económicos, promover la actividad empresarial, conciliar la vida familiar y laboral... destacando de todas ellas la promoción de una Formación Ocupacional que facilite la cualificación de las mujeres, para mejorar su acceso al mercado laboral. Esto quiere decir que, junto al desarrollo imprescindible de la legislación sociolaboral sobre la igualdad de hombres y mujeres (en relación a la custodia y cuidado de los hijos, la maternidad, subvenciones a la contratación...) hay que seguir incrementado la calidad del sistema formativo, ya que no sólo permite a la mujer su inserción en el mercado laboral, adaptando sus cualificaciones a las necesidades del mercado de trabajo, sino que también supone una mejora personal y social, una mejora integral de la persona.

Por tanto y a modo de síntesis, es imprescindible para el logro de estos objetivos trazar las siguientes líneas de acción, siguiendo a Silveira (2000):

- Estrechar los vínculos entre el sistema formativo y el sector productivo: definir los posibles nichos de empleo femenino existentes y abrir nuevos, actualizar sus perfiles ocupacionales...

- Sensibilizar a la sociedad y a sus actores para: apoyar y estimular un cambio en los patrones culturales y empresariales. 
- Desarrollar competencias claves para la empleabilidad de las mujeres. Entre ellas aparecen: competencias (básicas, transversales y actitudinales), elaboración de un proyecto personal viable de empleo y formación, diversificación de la participación femenina en la Formación Profesional, ofrecer mecanismos de apoyo económico y doméstico...

\section{Bibliografía.}

Arce, J.C. (1999). El trabajo de las mujeres en el derecho comunitario. Valencia: Tirant lo Blanch.

Battistone, L. (1992). El mercado de Trabajo y la Formación Profesional en las mujeres. En Ferrández, A. La formación ocupacional realidad y perspectivas. Madrid: Diagrama

Bianchi, M. (1994). Más allá del doble trabajo. En C. Borderías, C. Carrasco y C. Alemany, Las mujeres y el trabajo. Barcelona: Icaria.

CEDEFOP (1997). Evaluación de Programas europeos de formación, empleo y recursos humanos. Conferencia de Atenas, 1995. Salónica: CEDEFOP.

Chiva, I. (2003). Evaluación de programas de formación ocupacional en colectivos con riesgo de exclusión social. Tesis Doctoral. Universitat de València.

Chiva, I. (2005). Evaluación de los programas de formación ocupacional para mayores de 40 años. Revista Electrónica REDSI - Red Social Interactiva $n^{\circ}$ 6. Chiva, I. (2005). Consultado el 10 de enero de 2006 en http://redsirevista.cebses.org/index.asp?IdArt $=138$

Comisión Europea. (1996). Informe Especial: Nuevas oportunidades de empleo para las mujeres. Consultado el 10 de enero de 2006 en http://europa.eu.int/

Duru-Bellat, M. (1990). L'ecole des filles. Paris: L'Harmatan.

EUROSTAT (2000). Cifras Clave 1999. Consultado el 11 de febrero de 2006 en http://europa.eu.int/
González Hernández, A. y García Martínez, A. (1996). La formación y el empleo en las mujeres. En Educación, empleo y formación profesional. Actas del V Congreso Nacional de Educación Comparada. Valencia: Universitat de València.

Instituto Nacional de Estadística (2000). Encuesta de población activa 1999. Indicadores Sociales de España: Capítulo 4 Trabajo. Madrid: INE

Instituto Nacional de Estadística (2003). Indicadores Sociales de España 2003. Madrid: INE

Instituto Nacional de Estadística (2005). EPA II Trimestre 2005.Madrid: INE

Izquierdo, J. y otros (1988). La desigualdad de las mujeres en el uso del tiempo. Madrid. Ministerio de Asuntos Sociales. Instituto de la mujer.

Jornet, J.M. y Suárez, J.M. (1996a). Informe de Validación del Modelo de Evaluación EFO. Informe inédito, presentado ante la Conselleria de Trabajo y Asuntos Sociales de la Generalitat Valenciana.

Jornet, J.M. y Suárez, J.M. (1996b). Informe Síntesis del Modelo de Evaluación EFO. Informe inédito, presentado ante la Conselleria de Trabajo y Asuntos Sociales de la Generalitat Valenciana.

Jornet, J.M. y Suárez, J.M. (1996c). Informe de Perfiles y desarrollo de Estándares. Informe inédito, presentado ante la Conselleria de Trabajo y Asuntos Sociales de la Generalitat Valenciana.

Martin Arribas, J.J. (1993). El principio de igualdad de oportunidades entre hombres y mujeres a la luz del Derecho Comunitario Europeo. Revista de Estudios Europeos, 4.

ONU (1995). Cuarta Conferencia Mundial sobre las Mujeres. Beijing: ONU

Organización Internacional del Trabajo (1988). Mujer y trabajo, $n^{\circ} 2$. Ginebra: OIT.

Pearson, M. (1996). Experience, skill and competitiveness: the implications of an ageing population for the workplace. Luxembourg: Office for Official Publications of the European Communities.

Pedraza. B. (2001). La nueva formación profesional en España ¿Hacia un Sistema $\mathrm{Na}$ - 
cional de Cualificaciones Profesionales?. Consultado el 11 de enero de 2006 en http://www.cinterfor.es/

Perales, M.J. (2000). Enfoques de Evaluación de Formación Profesional y Continua. Estudio de Validación de un modelo. Tesis Doctoral. Universitat de València.

Perales, M.J. (2002). Enfoques de Evaluación de FPO. Estudio de Validación de un modelo. Valencia: SERVEF.

Pérez Carbonell, A. (1998). Metodología de la Evaluación de Programas: Evaluación de Programas de Postgrado en la Universitat de València. Publicada en Microficha por el Servei de Publicacions de la Universitat de València (2000).
Picchio, P. (1994) Mujeres. En Borderías, C. Carrasco, C. y Alemany, C. Las mujeres y el trabajo. Barcelona: Icaria.

Romans F. y Hardarson, O. (2005). Statistics in Focus 16/2005. EUROSTAT . Consultado el 10 de enero de 2006 en http://europa.eu.int/

Silveira, S. (2000). La dimensión de género en la formación y en las relaciones laborales. CINTERFOR/OIT. Consultado el 10 de enero de 2006 en: http://www.cinterfor.org/. Suárez, J.M. (1989). Proyecto Docente y de Investigación al Cuerpo de Titulares de Universidad. Universitat de Valencia: inédito, cortesía del autor.

\section{ABOUT THE AUTHORS / SOBRE LOS AUTORES}

Inmaculada Chiva (ichiva@uv.es). Profesora del Área de Métodos de Investigación y Diagnóstico en Educación de la Universidad de Valencia desde el año 2001. Sus principales líneas de trabajo van asociadas al Grupo GEM (www.uv.es/gem) al cual pertenece y son principalmente: Evaluación de la Formación Ocupacional y Continua en la Comunidad Valenciana, evaluación de la teleformación, estudios sobre la calidad de la Educación Primaria y Secundaria en la Comunidad Valenciana, formación de profesores, diseño y desarrollo de un banco de ítems, diseño de cuestionarios de contexto para la evaluación de sistemas educativos. Su dirección postal es: Facultad de Filosofía y CC. de la Educación. Avda. Blasco Ibáñez, 30 46010-Valencia (España). 
Chiva, I. (2006). Evaluación de los programas de formación ocupacional para el colectivo de mujeres.

RELIEVE, v. 12, n. 1, p. 49-74. http://www.uv.es/RELIEVE/v12n1/RELIEVEv12n1_3.htm

\section{ARTICLE RECORD / FICHA DEL ARTÍCULO}

\begin{tabular}{|c|c|}
\hline $\begin{array}{l}\text { Reference / } \\
\text { Referencia }\end{array}$ & $\begin{array}{l}\text { Chiva, Inmaculada (2006). Evaluación de los programas de formación ocupacional para el colectivo de } \\
\text { mujeres. Revista ELectrónica de Investigación y EValuación Educativa, v. 12, n. 1. } \\
\text { http://www.uv.es/RELIEVE/v12n1/RELIEVEv12n1_3.htm. Consultado en (poner fecha). }\end{array}$ \\
\hline Title / Título & $\begin{array}{l}\text { Evaluación de los programas de formación ocupacional para el colectivo de mujeres [Occupational } \\
\text { Training Evaluation Programs of women collective] }\end{array}$ \\
\hline $\begin{array}{l}\text { Authors / } \\
\text { Autores }\end{array}$ & Inmaculada Chiva \\
\hline $\begin{array}{l}\text { Review / } \\
\text { Revista }\end{array}$ & Revista ELectrónica de Investigación y EValuación Educativa (RELIEVE), v. 12, n. 1 \\
\hline ISSN & $1134-4032$ \\
\hline $\begin{array}{l}\text { Publication } \\
\text { date / } \\
\text { Fecha de } \\
\text { publicación }\end{array}$ & $\begin{array}{l}2006 \text { (Reception Date: } 2005 \text { February 12; Approval Date: } 2006 \text { April 2; Publication Date: } 2006 \\
\text { April 3) }\end{array}$ \\
\hline $\begin{array}{l}\text { Abstract / } \\
\text { Resumen }\end{array}$ & $\begin{array}{l}\text { This paper summarizes the results obtained from the evaluation of Occupational Training Programs } \\
\text { through which we checked the quality of service and the efficiency of the introduction of women into the } \\
\text { labour market, as well as the need to promote quality Occupational Training specifically for women that } \\
\text { will not only facilitate their introduction into the labour market by adapting their personal skills to its } \\
\text { needs, but will also encourage personal and social improvement.. } \\
\text { Este artículo trata de resumir los resultados obtenidos en la evaluación de los programas de Formación } \\
\text { Ocupacional para comprobar la calidad del servicio y la eficacia en el logro de la inserción laboral del } \\
\text { colectivo de mujeres. De esta manera, hemos comprobado la necesidad de seguir promoviendo una } \\
\text { Formación Ocupacional específica de calidad para la mujer ya que no sólo le va a permitir su inserción } \\
\text { en el mercado laboral, adaptando sus cualificaciones a las necesidades del mercado de trabajo, sino que } \\
\text { también le va a suponer una mejora personal y social. }\end{array}$ \\
\hline $\begin{array}{l}\text { Keywords } \\
\text { Descriptores }\end{array}$ & $\begin{array}{l}\text { Evaluation, occupational training, women, quality, labour market. } \\
\text { Evaluación, formación ocupacional, mujeres, calidad, inserción laboral }\end{array}$ \\
\hline \begin{tabular}{l|} 
Institution / \\
Institución
\end{tabular} & Universidad de Valencia (España) \\
\hline $\begin{array}{l}\text { Publication } \\
\text { site / } \\
\text { Dirección }\end{array}$ & http://www.uv.es/RELIEVE \\
\hline $\begin{array}{l}\text { Language / } \\
\text { Idioma }\end{array}$ & Spanish (Title, abstract and keywords in english ) \\
\hline
\end{tabular}

\section{Revista ELectrónica de Investigación y EValuación Educativa (RELIEVE)}

[ ISSN: 1134-4032 ]

(C) Copyright, RELIEVE. Reproduction and distribution of this articles it is authorized if the content is no modified and their origin is indicated (RELIEVE Journal, volume, number and electronic address of the document).

(C) Copyright, RELIEVE. Se autoriza la reproducción y distribución de este artículo siempre que no se modifique el contenido y se indique su origen (RELIEVE, volumen, número y dirección electrónica del documento). 